\title{
Evaluation of the anti-Thomsen-Friedenreich antibodies Nemod-TF1 and Nemod-TF2 as prognostic markers in breast cancer
}

\author{
Sabine Heublein ${ }^{1,2} \quad$ Markus Egger ${ }^{2,3} \cdot$ Junyan Zhu $^{2} \cdot$ Luisa Berger $^{2} \cdot$ Doris Mayr $^{4} \cdot$ Christian Schindlbeck $^{5}$. \\ Christina Kuhn ${ }^{2} \cdot$ Simone S. Hofmann ${ }^{2} \cdot$ Florian Schuetz $^{1} \cdot$ Udo Jeschke ${ }^{2,6} \cdot$ Nina Ditsch $^{2,6}$
}

\begin{abstract}
Purpose The TF (Thomsen-Friedenreich, CD176, Gal $\beta 1-3 \mathrm{GalNAc}$ ) carbohydrate moiety is known as a specific oncofetal carbohydrate epitope present in fetal and neoplastic tissue as well as in stem cells. TF was demonstrated to mediate tumorpromoting features and to be highly immunogenic. The current study aimed to evaluate whether presence of the TF antigen is associated with clinico-pathological parameters and prognosis of early breast cancer (BC).

Methods Primary BC tissue $(n=226)$ was stained for TF using two monoclonal anti-TF antibodies (Nemod-TF1, NemodTF2). Staining results were correlated to clinical data including survival.

Results Nemod-TF1 staining was positively correlated to lymph node metastasis $(p=0.03)$ and the presence of tumorassociated MUC1 (TA-MUC1; $p=0.003$ ). Further, the presence of the Nemod-TF1 epitope predicted worse prognosis in TA-MUC1 positive (overall survival: $p=0.026$ ) as well as in triple negative (overall survival: $p=0.002$; distant metastasisfree survival: $p=0.012) \mathrm{BC}$.

Conclusions The data presented here further support a role of TF in BC tumor biology. Whether anti-TF directed treatment approaches may gain clinical relevance in those cases determined as triple negative or TA-MUC1 positive remains to be determined.
\end{abstract}

Keywords Nemod-TF1/2 $\cdot$ CD176 $\cdot$ Breast cancer $\cdot$ Prognosis

\begin{tabular}{ll}
\multicolumn{2}{l}{ Abbreviations } \\
BC & Breast cancer \\
CIS & Carcinoma in situ \\
CSC & Cancer stem cell \\
DFS & Disease-free survival \\
DMFS & Distant metastasis-free survival \\
ER & Estrogen receptor \\
FFPE & Formalin-fixed, paraffin-embedded \\
IRS & Immunoreactive score \\
LRFS & Local recurrence-free survival \\
OS & Overall survival \\
PR & Progesterone receptor \\
REMARK & REporting recommendations for tumor \\
& MARKer prognostic studies
\end{tabular}

Sabine Heublein

sabine.heublein@med.uni-heidelberg.de

\author{
TA-MUC1 Tumor-associated mucin-1 \\ TF Thomsen-Friedenreich, CD176, \\ Galp1-3GalNAc \\ TNBC Triple negative BC \\ WHO World Health Organization
}

\section{Background}

The Thomsen-Friedenreich (TF, core-1) antigen is a specific oncofetal carbohydrate (Gal $\beta 1-3 \mathrm{GalNAc}$ ) moiety present on the surface of neoplastic cells of numerous cancer entities [1]. For instance, mucin-like glycoproteins such as MUC1 (CA15-3, CD227), which has developed to be the most widely used breast cancer (BC) serum tumor marker, were demonstrated to abundantly carry the TF antigen [2, $3]$. TF is covered by a cryptic glyco-structure in healthy adult tissue, but has been detected to become exclusively demasked during the process of malignant transformation [4]. Accordingly, TF is hardly found in non-neoplastic adult cells but widely present in cancer cells especially in 
epithelial descent. For this reason, TF has been hypothesized to be a tumor marker of exceptional specificity [4]. The TF carbohydrate moiety is linked to carrier molecules by $\alpha$ - or $\beta$-O-glycosylation and has therefore be termed as TF $\alpha$ or TF $\beta$, respectively [5]. As TF $\beta$ is absent in tumors of epithelial descent [5], in this manuscript the term TFunless otherwise stated-refers to $\mathrm{TF} \alpha$.

TF has been found to mediate endothelium adhesion and tumor invasion implying its potential as a marker of cancer cell aggressiveness [6]. A recent study of our group also demonstrated that the apoptotic potential of galectin-1 on BC cells is dependent on the presence of cell surface TF [7]. In addition, the TF glycoepitope is highly immunogenic and thus several attempts have been made to design antibodies targeting TF. These antibodies have already been shown to comprise anti-cancer cell activity in vitro and a mouse $\mathrm{BC}$ model $[8,9]$.

Producing monoclonal antibodies (mAbs) detecting $\mathrm{TF}$ appeared to be difficult in the past. In addition, mAbs detecting TF differ regarding specificity and selectivity and only few of them proved to be highly specific and selective for TF in its 'natural' context without showing cross-reactivity to synthetic TF-conjugates. Goletz et al. proposed Nemod-TF1 and Nemod-TF2 to be two of the most thoroughly characterized mAbs detecting TF [10]. Though basically Nemod-TF1 and Nemod-TF2 both detect the same carbohydrate moiety (i.e., TF), their fine specificities are different. Highly avid Nemod-TF2 detects TF but is also cross-reactive to $\operatorname{TF} \beta$ and to core-2, a carbohydrate structure similar but not equal to TF [10]. Since core-2 has not been found in humans and TF $\beta$ is not present in human tissue at a level detectable by IHC, the cross-reactivity of Nemod-TF2 to core- 2 does not alter the detection of TF by Nemod-TF2 in human tumors [10]. Nemod-TF1 holds both high affinity and specificity for the $\alpha$-anomer of TF (herein termed TF), is not cross-reactive to core-2, and even detects very low densities of TF. Therefore, NemodTF1 is regarded to be the most specific TF antibody which is seen as a basic requirement in terms of potentially using Nemod-TF1 as a therapeutic antibody in the future [10].

In view of the above, we selected the two highly selective TF antibodies Nemod-TF1 and Nemod-TF2 to investigate whether TF may be related to clinico-pathological parameters or prognosis of $\mathrm{BC}$ patients. To the best of our knowledge, no data exist upon Nemod-TF1 or NemodTF2 immunoreactivity from a large panel of BC patients. We thus correlated immunoreactivity of both Nemod-TF1 and Nemod-TF2 to clinico-pathological variables including overall survival (OS), local recurrence-free survival (LRFS), distant metastasis-free survival (DMFS), and disease-free survival (DFS).

\section{Methods}

\section{Patients and specimen characteristics}

Formalin-fixed, paraffin-embedded (FFPE) BC samples from 226 patients who underwent surgery from 1988 to 2000 due to a malignant tumor of the breast at the Department of Obstetrics and Gynecology, Ludwig-MaximiliansUniversity of Munich, Germany, were included in this study (Table 1). Histopathological and clinical data as well as the follow-up were retrieved from patients' charts, the laboratory archive, or from the Munich Cancer Registry. Study endpoints were defined as follows: $\mathrm{OS}=$ period of time from the date of surgery until the date of death or the date of last follow-up. Patients alive at the time of last follow-up and patients who died due to a non-BCrelated cause were treated as censored cases. $\mathrm{LRFS}=$ time a patient survives without developing local recurrence (i.e., ipsilateral breast or axilla); DMFS = time a patient survives without developing distant metastatic spread; DFS $=$ period a patient survives without local or distant evidence of disease [11]. Patients not having experienced an event (i.e., local recurrence [LRFS], distant metastasis [DMFS], either local or distant recurrence/metastasis [DFS]) were treated as censored cases.

\section{Assay methods}

\section{Immunohistochemistry (IHC)}

In total, 226 cases were investigated by Nemod-TF immunohistochemistry. Data on both antibody stainings were available from 222 cases. Four patient samples were only available for either Nemod-TF2 or Nemod-TF1 staining, respectively. The number of cases eligible for Nemod-TF1 IHC was 224. Nemod-TF2 was IHC was also performed in 224 cases.

IHC of TF and scoring was described by our group before $[12,13]$. Tissue samples were fixed in buffered formalin solution $(3.7 \%)$ immediately after resection and underwent standardized paraffin embedding. Samples were stored under standardized conditions. Tissue slides were cut using a microtome and dewaxed in xylene. Tissue peroxidase was blocked using 3\% peroxide in methanol. Following a descending series of alcohols, a washing and a blocking step slides were stained using Nemod-TF1 (mouse IgM, kappa produced by Glycotope, Berlin, Germany) or Nemod-TF2 (mouse IgM, kappa produced by Glycotope, Berlin, Germany) as described before [13, 14]. Antibody dilutions were 1:100 in case of Nemod-TF1 and 
Table 1 TF1 and TF2 positivities as correlated to clinico-pathological parameters

\begin{tabular}{|c|c|c|c|c|c|c|}
\hline & \multicolumn{3}{|c|}{ Nemod-TF1 } & \multicolumn{3}{|c|}{ Nemod-TF2 } \\
\hline & Neg. & Pos. & $p$ & Neg. & Pos. & $p$ \\
\hline \multicolumn{7}{|l|}{ Histology } \\
\hline NST & 75 & 54 & ns & 76 & 52 & $\mathrm{~ns}$ \\
\hline Non NST & 63 & 32 & & 53 & 43 & \\
\hline \multicolumn{7}{|l|}{ Subtype } \\
\hline Luminal A & 52 & 47 & ns & 51 & 49 & ns \\
\hline Luminal B & 50 & 21 & & 43 & 28 & \\
\hline Her2 pos. & 16 & 5 & & 12 & 9 & \\
\hline TNBC & 18 & 11 & & 22 & 7 & \\
\hline \multicolumn{7}{|l|}{ Grading } \\
\hline $\mathrm{G} 1, \mathrm{G} 2$ & 63 & 39 & ns & 59 & 42 & ns \\
\hline G3 & 29 & 23 & & 30 & 22 & \\
\hline \multicolumn{7}{|l|}{ pT } \\
\hline pT1 & 94 & 57 & ns & 88 & 65 & ns \\
\hline pT2-pT4 & 43 & 29 & & 40 & 30 & \\
\hline \multicolumn{7}{|l|}{$\mathrm{pN}$} \\
\hline pNO & 82 & 38 & 0.03 & 74 & 47 & ns \\
\hline pN1-pN3 & 50 & 43 & & 50 & 42 & \\
\hline \multicolumn{7}{|c|}{ CIS fraction in invasive CA } \\
\hline No & 67 & 38 & ns & 66 & 39 & ns \\
\hline Yes & 71 & 48 & & 63 & 56 & \\
\hline \multicolumn{7}{|l|}{ Age (years) } \\
\hline$\leq 60$ & 90 & 46 & ns & 85 & 52 & ns \\
\hline$>60$ & 48 & 39 & & 43 & 43 & \\
\hline
\end{tabular}

CIS carcinoma in situ in invasive CA, $n s$ not signficant
1:200 for Nemod-TF2. Following another series of washing steps biotinylated anti-IgM secondary antibody was applied and visualized using the Vectastain ${ }^{\circledR}$ Elite ABCKit (Vector Laboratories, Peterborough, GB) in combination with DAB as a chromogenic substrate. Slides were counterstained with hemalaun, washed, and mounted.

Ovarian cancer tissue served as positive control for Nemod-TF1 and Nemod-TF2 staining as explained elsewhere [12], while replacement of the primary antibody with mouse $\mathrm{IgM}$ was performed as negative control.

The immunoreactivity of Nemod-TF1 and Nemod-TF2 was examined by two independent observers by consensus. Samples were assessed by applying an established semi-quantitative immunoreactive score (IRS) [15-17]. The IR score quantifies immunoreactivity by multiplication of staining intensity (graded as $0=$ no, $1=$ weak, $2=$ moderate, and $3=$ strong staining) and percentage of positively stained cells $(0=$ no staining, $1=\leq 10 \%$ of the cells, $2=11-50 \%$ of the cells, $3=51-80 \%$ of the cells, and $4=\geq 81 \%$ of the cells). A Leitz (Wetzlar, Germany) microscope was employed, and representative images were taken by a CCD color camera (JVC, Japan). Since both Nemod-TF1 and Nemod-TF2 positivity rates were found to be quite low; the threshold was set at an IR score of 0 with cases scored as IRS higher than 0 counted as positive. TA-MUC1 staining on the study panel has been published by our group before [18].

\section{Statistical analysis methods}

This study has been carried out according to the REMARK (REporting recommendations for tumor MARKer prognostic studies) criteria [19]. We used the IBM statistic package SPSS (version 24) and Microsoft Excel 2010 to test data for statistical significance and to plot graphs. Chi-square test was performed to test data for statistical independence. Fisher's exact test was used where numbers in each group were insufficient for Chi-square test. Survival analysis was done by applying the log rank test and data are presented as Kaplan-Meier survival curves. Observations with $p<0.05$ were considered as statistically significant.

\section{Results}

\section{Study cohort}

A breast tumor smaller than $2 \mathrm{~cm}$ in size was diagnosed in $68.0 \%$ of cases $(n$ (data available $)=225, n(\mathrm{pT} 1)=153$ 
$(68.0 \%), n(\mathrm{pT} 2)=66(29.3), n(\mathrm{pT} 3)=1(0.4 \%), n(\mathrm{pT} 4)=5$ $(2.2 \%))$, and about half of all patients were diagnosed for cancer without lymph node metastasis ( $n$ (data available $)=215 ; n(\mathrm{pN} 0)=122(56.7 \%))$, respectively. About one-third of tumors was classified as high grade $(n(\mathrm{G} 3)=53$ $(34.2 \%))$. A significant number of cases also displayed a DCIS/LCIS fraction within the invasive carcinomas. Information on intrinsic subtypes was available from 222 cases and subtypes were distributed as follows: $n$ (Luminal $\mathrm{A})=100(45.0 \%), n($ Luminal B $)=71(32.0 \%), n($ Her 2 positive $)=21(9.5 \%), n($ TNBC $)=30(13.5 \%)$. Study endpoints were OS, DMFS, DFS, and LRFS.

Mean OS was 12.2 years (95\% CI 11.6-12.8 years), mean follow-up was 9.8 years (95\% CI 9.27-10.3 years), and 49 cancer-related deaths were documented. Another 23 women died due to non-cancer-related reasons and were treated as censored cases. Mean DMFS was 13.0 years $(n$ (events) $=34)$, mean DFS was 11.4 years $(n$ (events $)=55)$, and mean LRFS was 13.4 years $(n$ (events $)=32$ ). Mean patient age was $58.3 \pm 13.3$ years. Further patients' characteristics are listed in Table 1.

\section{TF with respect to clinico-pathological parameters}

Both Nemod-TF1 and Nemod-TF2 showed immunopositivity in about $40 \%$ of cases (Nemod-TF1: $38.4 \%$, NemodTF2: 42.4\%; Fig. 1). Correlation analysis revealed a strong concordance between Nemod-TF1 and Nemod-TF2 staining $(p<0.001)$ which was independent of the intrinsic BC subtype (i.e., Luminal A/B, Her2 positive, TNBC), respectively. Co-expression of both Nemod-TF epitopes was observed in 63 out of 222 cases ('double positive', 28.4\%), while the absence of both epitopes was found in 105 cases ('double negative,' 47.3\%) samples. The presence of neither NemodTF1 nor Nemod-TF2 epitope was related to histopathological parameters like histology, histologic subtype, pT stage, the presence of an in situ component, or tumor grade (Table 1). TF immunostaining was not associated with patient age. Those cases that presented with lymph node metastasis at initial diagnosis expressed TF significantly more often than lymph node-negative tumors did (fraction of Nemod-TF1 positive in $\mathrm{pN}+: 46.2 \%$ vs. fraction of Nemod-TF1 positive in pN0: $31.7 \% ; p=0.03)$. Nemod-TF1 was inversely correlated to anthracycline-containing systemic treatment $(p=0.048)$ while Nemod-TF2 was positively correlated with radiotherapy ( $p=0.047$; Table 2$)$.

Tumors expressing the Gatipotuzumab (formerly known as PankoMabGEX) epitope (=TA-MUC1) at the tumor cell membrane stained positive for Nemod-TF1 more often than those classified as negative for TA-MUC1 (fraction of Nemod-TF1 positive in TA-MUC1 positive: $44.9 \%$ vs. fraction of Nemod-TF1 positive in TA-MUC1 negative: $23.5 \%$; $p=0.003)$. The presence of the Nemod-TF1 epitope was inversely correlated to ki67 ( $p=0.013$ ). Immunoreactivity of Nemod-TF2 was significantly higher in ER-positive (fraction of Nemod-TF2 positive in ER positive: $46.0 \%$ vs. fraction of Nemod-TF2 positive in ER negative: $20.7 \%$; $p=0.011$ ) and PR-positive cancers (fraction of Nemod-TF2 positive in PR positive: $46.9 \%$ vs. fraction of Nemod-TF2 positive in PR negative: $31.7 \%$; $p=0.049)$. Finally, Nemod-TF2 was positively correlated to membrane staining of TA-MUC1, too (fraction of Nemod-TF2 positive in TA-MUC1 positive: $49.0 \%$ vs. fraction of Nemod-TF2 positive in TA-MUC1 negative: $27.5 \% ; p=0.003$ ) (Table 3 ).

\section{Nemod-TF1 immunoreactivity predicts shortened distant metastasis-free and OS in triple negative BC patients}

BC samples were stratified as either Nemod-TF1 positive vs. negative or Nemod-TF2 positive vs. negative, respectively. Neither immunoreactivity of Nemod-TF1 nor immunoreactivity of Nemod-TF2 was associated with OS, LRFS, DMFS, or DFS regarding the non-stratified study cohort. Then, immunopositivity of both anti-TF antibodies was tested for its ability to predict prognosis in subgroups of the cohort. Staining of Nemod-TF1 was related to shortened OS in cases expressing the carbohydrate
Fig. 1 Staining of TF by Nemod-TF1 and Nemod-TF2 in BC tissue. Representative photomicrographs of Nemod-TF1 and Nemod-TF2 immunostaining are presented $(25 \times$ lens $)$
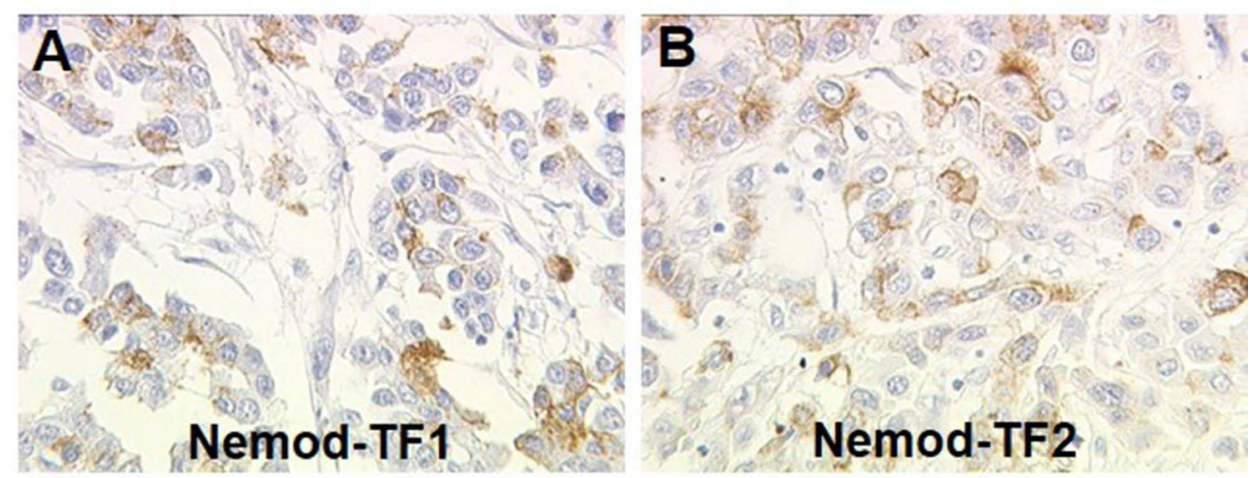
Table 2 Local and systemic treatment of patients studied

\begin{tabular}{|c|c|c|c|c|c|c|c|c|c|}
\hline & Data availabl & & & Nemo & TF1 & & Nemo & TF2 & \\
\hline & Nemod-TF1 & Nemod-TF2 & & Neg. & Pos. & & Neg. & Pos. & \\
\hline Type of breast surgery & 185 & 185 & Breast conserving & 88 & 49 & ns & 84 & 55 & ns \\
\hline & & & Mastectomy & 28 & 20 & & 25 & 21 & \\
\hline ALNE & 175 & 175 & Performed & 113 & 62 & & 106 & 69 & \\
\hline Radiation & 137 & 139 & Yes & 87 & 43 & ns & 81 & 51 & 0.047 \\
\hline & & & No & 6 & 1 & & 7 & 0 & \\
\hline Systemic treatment & 105 & 106 & No & 40 & 26 & ns & 40 & 27 & ns \\
\hline & & & Yes & 27 & 12 & & 25 & 14 & \\
\hline Anthracycline-containing CHT & 104 & 105 & No & 47 & 34 & 0.048 & 47 & 35 & ns \\
\hline & & & Yes & 19 & 4 & & 17 & 6 & \\
\hline Platinum-containing CHT & 104 & 105 & No & 64 & 37 & ns & 62 & 40 & ns \\
\hline & & & Yes & 2 & 1 & & 2 & 1 & \\
\hline Taxane-containing $\mathrm{CHT}$ & 104 & 105 & No & 55 & 35 & ns & 52 & 39 & ns \\
\hline & & & Yes & 11 & 3 & & 12 & 2 & \\
\hline Endocrine treatment & 105 & 106 & No & 22 & 9 & ns & 22 & 10 & ns \\
\hline & & & Yes & 45 & 29 & & 43 & 31 & \\
\hline
\end{tabular}

$A L N E$ axillary lymphonodectomy, $n s$ not significant, neg. negative, pos. positive, $C H T$ chemotherapy

Table 3 Nemod-TF1 and Nemod-TF2 positivities as correlated to cancer biomarkers

\begin{tabular}{|c|c|c|c|c|c|c|}
\hline & \multicolumn{3}{|c|}{ Nemod-TF1 } & \multicolumn{3}{|c|}{ Nemod-TF2 } \\
\hline & Neg. & Pos. & $p$ & Neg. & Pos. & $p$ \\
\hline \multicolumn{7}{|l|}{ ER } \\
\hline Negative & 19 & 10 & ns & 23 & 6 & 0.011 \\
\hline Positive & 105 & 68 & & 94 & 80 & \\
\hline \multicolumn{7}{|l|}{ PR } \\
\hline Negative & 39 & 21 & ns & 41 & 19 & 0.049 \\
\hline Positive & 74 & 53 & & 68 & 60 & \\
\hline \multicolumn{7}{|l|}{ Her2 } \\
\hline Negative & 98 & 62 & ns & 97 & 62 & ns \\
\hline Positive & 16 & 3 & & 11 & 7 & \\
\hline \multicolumn{7}{|l|}{ ki67 } \\
\hline Negative & 52 & 48 & 0.013 & 52 & 49 & ns \\
\hline Positive & 51 & 21 & & 44 & 28 & \\
\hline \multicolumn{7}{|c|}{ Gatipotuzumab $^{\text {mem }}$} \\
\hline Negative & 52 & 16 & 0.003 & 50 & 19 & 0.003 \\
\hline Positive & 86 & 70 & & 79 & 76 & \\
\hline \multicolumn{7}{|c|}{ Gatipotuzumab $^{\text {cyt }}$} \\
\hline Negative & 94 & 52 & $\mathrm{~ns}$ & 87 & 58 & ns \\
\hline Positive & 44 & 34 & & 42 & 37 & \\
\hline
\end{tabular}

$n s$ not significant

epitope TA-MUC1 at the cell surface (as detected by Gatipotuzumab) (OS: $p=0.026$, Fig. 2c). Regarding DMFS ( $p=0.057$, Fig. 2a) and DFS ( $p=0.067$, Supp. Fig. 1a), this observation was of borderline significance. DMFS and OS of patients diagnosed for triple negative BC were significantly shortened in case of expressing the Nemod-TF1 epitope (DMFS: $p=0.012$, Fig. $2 b$; OS: $p=0.002$, Fig. $2 \mathrm{~d})$. This association failed to be significant $(p=0.091$, Supp. Fig. 1b) for DFS. Multivariate models were built to test whether Nemod-TF1 might evolve to be an independent prognosticator in the subgroups mentioned above. Nemod-TF1 proved to be an independent predictor for OS and DMFS in TNBC (Table 4). Since data on systemic treatment were available from 17 TNBC patients 


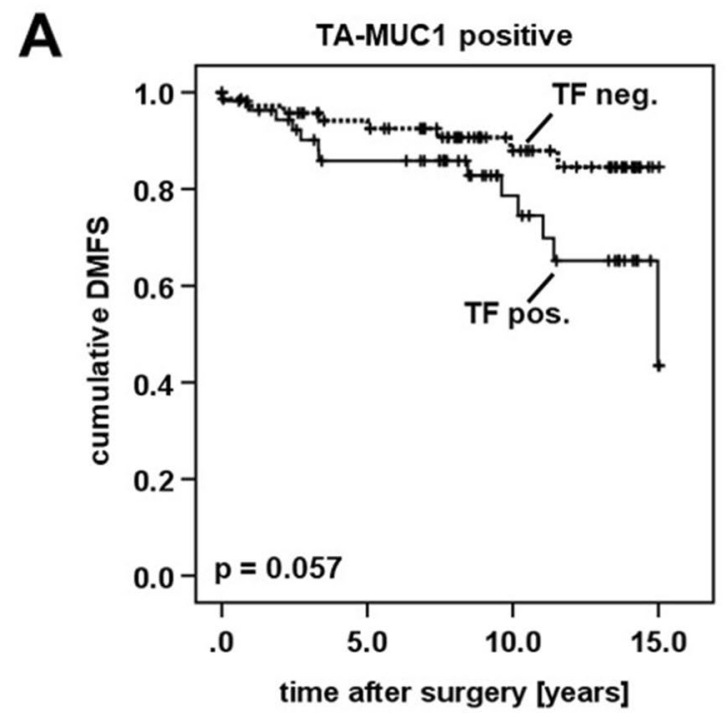

B
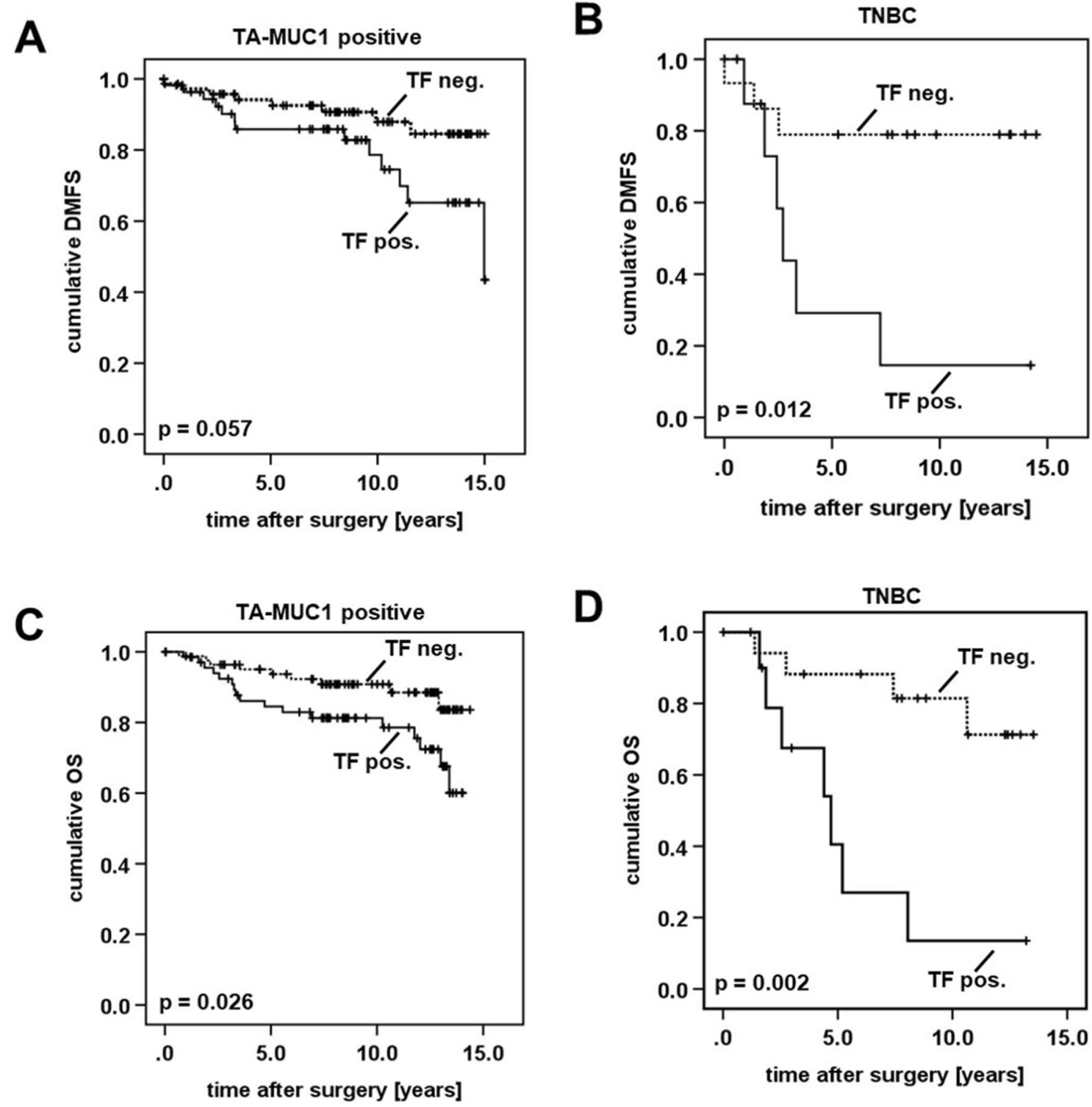

D

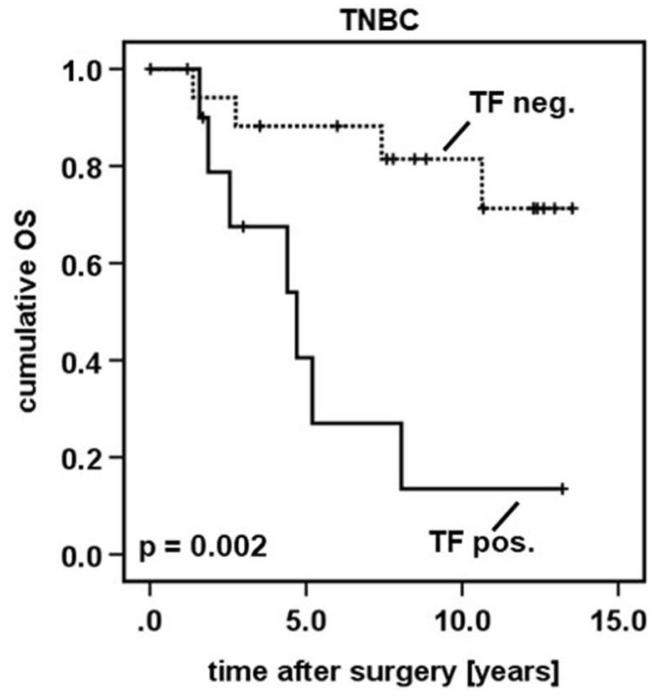

Fig. 2 Presence of the Nemod-TF1 epitope predicts shortened metastasis-free (DMFS) and OS in subgroup analysis. TF as detected by Nemod-TF1 was found to be predictive for shortened OS in a subgroup of breast cancers expressing the Gatipotuzumab epitope TA-

only, just type of breast surgery, tumor size, lymph node status, patient age, and Nemod-TF1 staining were included in the multivariate model.

On the opposite, though not reaching statistical significance, the presence of TF, as detected by Nemod-TF1, seemed to be associated with favorable DMFS in Her2-positive cases $(p=0.057$; Supplementary Fig. 2).

\section{Discussion}

This study detected immunoreactivity of Nemod-TF1 to predict prognosis in $\mathrm{BC}$ cases classified as either positive for the Gatipotuzumab epitope TA-MUC1 (membrane staining) or

as triple negative.

MUC1 at the cell surface (TA-MUC1 ${ }^{\text {mem }}$ ) (c). Regarding DMFS, this association was of borderline significance only (a). In addition, expression of the Nemod-TF1 epitope was predictive for shortened DMFS (b) and OS (d) in triple negative breast cancer 
Table 4 Multivariate Cox regression analysis in TNBC

\begin{tabular}{|c|c|c|c|c|}
\hline & \multirow[t]{2}{*}{$\operatorname{Exp}(B)$} & \multirow[t]{2}{*}{$p$} & \multicolumn{2}{|c|}{$\begin{array}{l}95.0 \% \text { CI for } \\
\operatorname{exp(B)}\end{array}$} \\
\hline & & & Lower & Upper \\
\hline \multicolumn{5}{|l|}{ Distant metastasis-free survival } \\
\hline pT (T1 vs. T2/3/4) & 0.588 & 0.544 & 0.105 & 3.275 \\
\hline pN (neg. vs. pos.) & 13.486 & 0.010 & 1.886 & 96.427 \\
\hline Age ( $\leq 60$ years vs. $>60$ years $)$ & 0.173 & 0.136 & 0.017 & 1.736 \\
\hline Breast surgery (con. vs. ME) & 1.010 & 0.934 & 0.796 & 1.282 \\
\hline Nemod-TF1 (neg. vs. pos.) & 7.604 & 0.038 & 1.123 & 51.506 \\
\hline \multicolumn{5}{|l|}{ Overall survival } \\
\hline pT (T1 vs. T2/3/4) & 0.342 & 0.243 & 0.056 & 2.074 \\
\hline pN (neg. vs. pos.) & 6.915 & 0.025 & 1.276 & 37.487 \\
\hline Age ( $\leq 60$ years vs. $>60$ years $)$ & 0.068 & 0.025 & 0.007 & 0.719 \\
\hline Breast surgery (con. vs. ME) & 1.022 & 0.049 & 1.000 & 1.045 \\
\hline Nemod-TF1 (neg. vs. pos.) & 11.929 & 0.002 & 2.500 & 56.916 \\
\hline
\end{tabular}

Con breast conserving, $M E$ mastectomy

Immunopositivity of either Nemod-TF1 or Nemod-TF2 was present in about $40 \%$ of BC samples. In line with this, others have demonstrated a similar fraction of TF-positive cases in different cancer entities and report comparable staining patterns $[4,20,21]$. Since TF has been hypothesized to be an oncofetal carbohydrate tumor marker expressed on cancer stem cells (CSC) or cell populations directly descending from them, such a distribution pattern may be rather expected [4]. Thus, to get a representative impression of Nemod-TF1/2 staining, IHC analysis presented here was performed on slides cut from tumor paraffine blocks rather than from core biopsies of tissue microarrays. We observed a strong positive correlation between Nemod-TF1 and NemodTF2 staining $(p<0.001)$. Although there was a positive correlation of the two antibodies, they differed regarding their correlation to clinico-pathological parameters and prognosis prediction. We hypothesize this to be due to the different fine specificity and affinity of the two as explained in the "Background" section.

TF was shown to be linked to CSC markers (e.g., CD44) by $O$-glycosylation [22]. Supporting its features as a CSC marker, TF has been highlighted to be abundantly present on circulating tumor cells [23] and to promote metastatic spread [8]. Gunkel et al. used A78-G/A7 to stain tissue samples of mucinous BC and their corresponding lymph node metastasis for TF. They found a strong co-incidence of TF expression in primary mucinous BC samples and lymph node metastasis [24]. We also report a positive correlation of TF expression and the presence of lymph node metastasis. These results may imply that the presence of the TF antigen in the primary breast tumor may facilitate metastatic spread to axillary lymph nodes or distant sites during early carcinogenesis. A biomarker reliably predicting lymph node positivity in patients diagnosed for primary BC may become of particular importance in terms of differentiating those patients with high vs. low risk for cancer spread to lymph nodes. While lymph node dissection may not be necessary in patients with low risk for positive lymph nodes, it may remain to be an indispensable part of surgical treatment of patients at high risk. Hence, a biomarker predicting lymph node status may assist to safely de-escalate surgical radicality. About half of all cases with positive axillary lymph nodes expressed the Nemod-TF1 epitope (fraction of Nemod-TF1 positive in $\mathrm{pN}+: 46.2 \%)$. However, 38 (31.7\%) out of 120 patients diagnosed as lymph node negative were found to express the epitope of Nemod-TF1, too. As a consequence, though expression of the Nemod-TF1 epitope significantly correlates with lymph node positivity, it seems to too unspecific to predict the lymph node status as a single marker. Thus, to clear whether a biomarker set including Nemod-TF1 may assist to prognosticate cancer spread to axillary lymph nodes, a specifically designed and powered trial would need to be set up.

Up to now, several glycoproteins in particular those classified as mucin-like glycoproteins have been identified to act as TF carrier molecules $[4,22]$. We performed correlation analysis thus to screen for potential candidates. Statistical correlation in our study sample confirms that membranous TA-MUC1 (as detected by Gatipotuzumab) carries TF and exposes it on the cell surface [25]. In line with this, canonical MUC1 has already been demonstrated to get glyco-modified by the TF moiety in breast and gastric cancer [26, 27]. MUC1 itself holds kinase-like activities [28], may directly modulate downstream kinase signaling [29], and has been demonstrated to regulate multiple tumor biologic features [29]. Interestingly, the presence of TF predicted shortened DDFS and OS especially in the TA-MUC1-positive subgroup, supporting that the oncogenic activity of TF may somehow be dependent on expression of TA-MUC1 at the cell membrane. Insight analysis apart from statistical correlation is indispensable in order to prove this.

TF has been described in multiple types of cancer. However, whether the presence of TF may be related to patients' prognosis remains to be contradictory. Though the majority of studies published on this topic report TF to be associated with unfavorable prognosis [30-33], others found TF to be a positive predictor [23,34]. Despite appearing inconsistent or even conflicting, both statements may be interpreted by TF exerting different biological effects in BC, i.e., alteration of the host anti-tumor immune response and direct regulation of cell signaling. In detail, since TF-which has been named the 'hidden tumor antigen' [10]—is covered by a "glycocap" in non-neoplastic tissue and becomes exposed only during malignant transformation [4], it may be recognized as a foreign, 'non-self' antigen by the host's immune system. Therefore, TF might facilitate a kind of host anti-tumor 
immune response enabling the host to initiate a more effective anti-tumor immune defense [23]. As a consequence, those cancer cells that expose TF on their surface may get eliminated more easily than those cancer cells not expressing TF. This seems to be especially attractive in terms of developing anti-cancer, i.e., anti-TF-directed vaccines or therapeutic antibodies for personalized oncology. Bearing in mind its immunogenic, 'non-self' characteristics, the presence of the TF epitope may be causally related to favorable DDFS in Her2-positive BC patients by augmenting a host anti-tumor immune action. Though this hypothesis seems to be conclusive and our analysis demonstrated survival curves of TF-positive vs. TF-negative patients to be clearly separated in Her2-positive BC, this did not reach statistical significance-potentially due to the small number of patients in this subgroup. Finally, it needs to be mentioned that this was a retrospective study and that patients were treated between 1988 and 2000, i.e., before the trastuzumab era. Whether our results can be reproduced in patients non-naïve for trastuzumab remains to be analyzed.

On the other side, antibodies targeting TF effectively blocked cell proliferation and extended survival of mice with metastatic $\mathrm{BC}[8,9,35]$, hence supporting the hypothesis that $\mathrm{TF}$ itself may comprise pro-tumorigenic actions and may thus be associated with worse prognosis. This seems to turn especially obvious in those $\mathrm{BC}$ cases either positive for the Gatipotuzumab epitope TA-MUC1 at the cell membrane or classified as triple negative. TA-MUC1 functions as a TF carrier molecule and — as speculated above-might be involved in mediating TF oncogenic activity. To gain mechanistic insights, functional studies using the NemodTF1 antibody would be required. Regarding TNBC, the presence of Nemod-TF1 was prognostic for shortened OS and DMFS within both uni- and multivariate analysis. However, results need to be interpreted with care since herein only relatively few TNBC cases were studied. Larger trials are needed to validate these results and to verify whether $\mathrm{TF}$ may turn out to be a targetable epitope in BC. Especially in case of TNBC, there is an unmet clinical need to develop effective therapies to target molecules that-like $\mathrm{TF}$-are widely expressed in TNBC and that potentially hold oncogenic activity in this molecular subtype.

Acknowledgements The authors thank Irmgard Wiest and Susanne Kunze $(\dagger)$ for their excellent technical assistance. We would further like to acknowledge Jutta Engel and Max Wiedemann for their help with collecting follow-up data from the Munich Cancer Registry. Parts of this work are part of ME's MD thesis obtained at the Department of Obstetrics and Gynecology, LMU Munich, Germany [36].

Author contributions SH participated in design and coordination of the study, significantly participated in experimental assays and analysis as well as in statistical analysis, and wrote the manuscript. ME participated in IHC assays and analysis and helped to draft the manuscript. LB participated in data collection and analysis. JZ participated in experimental assays. DM supervised IHC as a gynecologic pathologist and participated in IHC analysis. CS participated in the design of the study and carefully reviewed the manuscript for important intellectual content. CK optimized Nemod-TF staining and participated in immunohistochemistry. SSH participated in cell culture experiment design and conduction. FS helped to draft the manuscript and revised the manuscript for important intellectual content. UJ conceived and coordinated the study, participated in study design, and approved the final version of the manuscript. ND conceived and coordinated the study, participated in study design, and approved the final version of the manuscript.

Funding The study was financed by the Department of Obstetrics and Gynecology, Ludwig-Maximilians-University of Munich, Munich, Germany. No special funding was received.

\section{Compliance with ethical standards}

Conflict of interest All authors read the manuscript and agree to the publication of the manuscript. SH reports grants from the following organizations/companies: FöFoLe LMU Munich Medical Faculty, FERRING, Novartis Oncology, Astra Zeneca, Apceth, Heuer Stiftung, Deutsche Forschungsgemeinschaft. She further reports personal fees from Roche and non-financial support from Addex. ND held honorary speeches for Roche, AstraZeneca, Mentor, Omniamed, TEVA, and MSD. All the support listed here has been received outside the submitted work. Remaining authors have no competing interests to declare.

Ethical approval This article does not contain any studies with animals performed by any of the authors. All procedures performed in studies involving human participants were in accordance with the ethical standards of the institutional and/or national research committee and with the 1964 Helsinki declaration and its later amendments or comparable ethical standards. The tissue samples were retrieved from the archive of Obstetrics and Gynecology, Ludwig-Maximilians-University, Munich, Germany. The study was approved by the Ethics Committee of the Ludwig-Maximilians-University, Munich, Germany (approval number: 18-166). The tumor tissue re-used for our analysis had initially been collected for histopathological diagnostics. At the time, the tissue was examined for the current study all diagnostic procedures had already been fully completed and thus the tissue was classified as "leftover" material. Clinical data and tissue specimens were irreversibly anonymized prior to inclusion in the study. Researchers were blinded from patient data during experimental and statistical analysis.

Informed consent As per declaration of our ethics committee [Ludwig-Maximilians-University, Munich, Germany (approval number: 18-166)], no written informed consent of the participants is needed given the circumstances described above.

\section{References}

1. Karsten U, Goletz S (2015) What controls the expression of the core-1 (Thomsen-Friedenreich) glycotope on tumor cells? Biochemistry (Mosc) 80(7):801-807. https://doi.org/10.1134/S0006 297915070019

2. Dippold W, Steinborn A, Meyer zum Buschenfelde KH (1990) The role of the Thomsen-Friedenreich antigen as a tumor-associated molecule. Environ Health Perspect 88:255-257

3. Satyanarayana J, Gururaja TL, Narasimhamurthy S, Naganagowda GA, Levine MJ (2001) Synthesis and conformational features of human salivary mucin C-terminal derived peptide epitope 
carrying Thomsen-Friedenreich antigen: implications for its role in self-association. Biopolymers 58(5):500-510. https:// doi.org/10.1002/1097-0282(20010415)58:5\%3c500:AID-BIP10 25\%3e3.0.CO; $2-5$

4. Karsten U, Goletz S (2013) What makes cancer stem cell markers different? SpringerPlus 2(1):301. https://doi. org/10.1186/2193-1801-2-301

5. Axford SE (2003) Glycobiology and Medicine, 1st edn. Springer, New York

6. Yu LG, Andrews N, Zhao Q, McKean D, Williams JF, Connor LJ, Gerasimenko OV, Hilkens J, Hirabayashi J, Kasai K, Rhodes JM (2007) Galectin-3 interaction with Thomsen-Friedenreich disaccharide on cancer-associated MUC1 causes increased cancer cell endothelial adhesion. J Biol Chem 282(1):773-781. https://doi. org/10.1074/jbc.M606862200

7. Geiger P, Mayer B, Wiest I, Schulze S, Jeschke U, Weissenbacher $\mathrm{T}$ (2016) Binding of galectin-1 to breast cancer cells MCF7 induces apoptosis and inhibition of proliferation in vitro in a $2 \mathrm{D}$ and 3D- cell culture model. BMC Cancer 16(1):870. https://doi. org/10.1186/s12885-016-2915-8

8. Rittenhouse-Olson K (2007) Jaa-f11: extending the life of mice with breast cancer. Expert Opin Biol Ther 7(7):923-928. https:// doi.org/10.1517/14712598.7.7.923

9. Ferguson K, Yadav A, Morey S, Abdullah J, Hrysenko G, Eng JY, Sajjad M, Koury S, Rittenhouse-Olson K (2014) Preclinical studies with JAA-F11 anti-Thomsen-Friedenreich monoclonal antibody for human breast cancer. Future Oncol 10(3):385-399. https://doi.org/10.2217/fon.13.209

10. Goletz S, Cao Y, Danielczyk A, Ravn P, Schoeber U, Karsten U (2003) Thomsen-Friedenreich antigen: the "hidden" tumor antigen. Adv Exp Med Biol 535:147-162

11. He ZY, Wu SG, Zhou J, Li FY, Lin Q, Lin HX, Sun JY (2015) Postmastectomy radiotherapy improves disease-free survival of high risk of locoregional recurrence breast cancer patients with T1-2 and 1 to 3 positive nodes. PLoS ONE 10(3):e0119105. https ://doi.org/10.1371/journal.pone.0119105

12. Heublein S, Page SK, Mayr D, Ditsch N, Jeschke U (2016) p53 determines prognostic significance of the carbohydrate stem cell marker TF1 (CD176) in ovarian cancer. J Cancer Res Clin Oncol 142(6):1163-1170. https://doi.org/10.1007/s00432-016-2126-3

13. Jeschke U, Walzel H, Mylonas I, Papadopoulos P, Shabani N, Kuhn C, Schulze S, Friese K, Karsten U, Anz D, Kupka MS (2009) The human endometrium expresses the glycoprotein mucin- 1 and shows positive correlation for Thomsen-Friedenreich epitope expression and galectin-1 binding. J Histochem Cytochem 57(9):871-881. https://doi.org/10.1369/jhc.2009.952085

14. Engelstaedter V, Fluegel B, Kunze S, Mayr D, Friese K, Jeschke U, Bergauer F (2012) Expression of the carbohydrate tumour marker Sialyl Lewis A, Sialyl Lewis X, Lewis Y and ThomsenFriedenreich antigen in normal squamous epithelium of the uterine cervix, cervical dysplasia and cervical cancer. Histol Histopathol 27(4):507-514

15. Dian D, Janni W, Kuhn C, Mayr D, Karsten U, Mylonas I, Friese K, Jeschke U (2009) Evaluation of a novel anti-mucin 1 (MUC1) antibody (PankoMab) as a potential diagnostic tool in human ductal breast cancer; comparison with two established antibodies. Onkologie 32(5):238-244. https://doi.org/10.1159/000209280

16. Dian D, Lenhard M, Mayr D, Heublein S, Karsten U, Goletz S, Kuhn C, Wiest I, Friese K, Weissenbacher T, Jeschke U (2013) Staining of MUC1 in ovarian cancer tissues with PankoMabGEX detecting the tumour-associated epitope, TA-MUC1, as compared to antibodies HMFG-1 and 115D8. Histol Histopathol 28(2):239-244

17. Scholz C, Toth B, Barthell E, Mylonas I, Weissenbacher T, Friese K, Jeschke U (2009) Immunohistochemical expression of glycodelin in breast cancer correlates with estrogen-receptor alpha and progesterone-receptor a positivity. Histol Histopathol 24(4):467-471

18. Heublein S, Mayr D, Egger M, Karsten U, Goletz S, Angele M, Gallwas J, Jeschke U, Ditsch N (2015) Immunoreactivity of the fully humanized therapeutic antibody PankoMab-GEX is an independent prognostic marker for breast cancer patients. J. Exp. Clin. Cancer Res 34:50. https://doi.org/10.1186/s1304 6-015-0152-7

19. McShane LM, Altman DG, Sauerbrei W, Taube SE, Gion M, Clark GM (2005) Reporting recommendations for tumor marker prognostic studies. J Clin Oncol 23(36):9067-9072. https://doi. org/10.1200/JCO.2004.01.0454

20. Cao Y, Karsten UR, Liebrich W, Haensch W, Springer GF, Schlag PM (1995) Expression of Thomsen-Friedenreich-related antigens in primary and metastatic colorectal carcinomas. A reevaluation. Cancer 76(10):1700-1708

21. Imai J, Ghazizadeh M, Naito Z, Asano G (2001) Immunohistochemical expression of $\mathrm{T}$, Tn and sialyl-Tn antigens and clinical outcome in human breast carcinoma. Anticancer Res 21(2B):1327-1334

22. Lin WM, Karsten U, Goletz S, Cheng RC, Cao Y (2011) Expression of CD176 (Thomsen-Friedenreich antigen) on lung, breast and liver cancer-initiating cells. Int J Exp Pathol 92(2):97-105. https://doi.org/10.1111/j.1365-2613.2010.00747.x

23. Schindlbeck C, Jeschke U, Schulze S, Karsten U, Janni W, Rack B, Krajewski S, Sommer H, Friese K (2007) Prognostic impact of Thomsen-Friedenreich tumor antigen and disseminated tumor cells in the bone marrow of breast cancer patients. Breast Cancer Res Treat 101(1):17-25. https://doi.org/10.1007/s1054 9-006-9271-3

24. Gunkel L, Mylonas I, Richter DU, Makovitzky J (2005) Immunohistochemical studies of mucinous mammary carcinomas and their metastases. Anticancer Res 25(3A):1755-1759

25. Danielczyk A, Stahn R, Faulstich D, Loffler A, Marten A, Karsten U, Goletz S (2006) PankoMab: a potent new generation anti-tumour MUC1 antibody. Cancer Immunol Immunother 55(11):1337-1347. https://doi.org/10.1007/s00262-006-0135-9

26. Masuzawa Y, Miyauchi T, Hamanoue M, Ando S, Yoshida J, Takao S, Shimazu H, Adachi M, Muramatsu T (1992) A novel core protein as well as polymorphic epithelial mucin carry peanut agglutinin binding sites in human gastric carcinoma cells: sequence analysis and examination of gene expression. $\mathrm{J}$ Biochem 112(5):609-615

27. Lloyd KO, Burchell J, Kudryashov V, Yin BW, Taylor-Papadimitriou J (1996) Comparison of O-linked carbohydrate chains in MUC-1 mucin from normal breast epithelial cell lines and breast carcinoma cell lines. Demonstration of simpler and fewer glycan chains in tumor cells. J Biol Chem 271(52):33325-33334

28. Quin RJ, McGuckin MA (2000) Phosphorylation of the cytoplasmic domain of the MUC1 mucin correlates with changes in cellcell adhesion. Int J Cancer 87(4):499-506

29. Raina D, Kharbanda S, Kufe D (2004) The MUC1 oncoprotein activates the anti-apoptotic phosphoinositide 3-kinase/ Akt and Bcl-xL pathways in rat $3 \mathrm{Y} 1$ fibroblasts. J Biol Chem 279(20):20607-20612. https://doi.org/10.1074/jbc.M310538200

30. Baldus SE, Zirbes TK, Glossmann J, Fromm S, Hanisch FG, Monig SP, Schroder W, Schneider PM, Flucke U, Karsten U, Thiele J, Holscher AH, Dienes HP (2001) Immunoreactivity of monoclonal antibody BW835 represents a marker of progression and prognosis in early gastric cancer. Oncology 61(2):147-155. https://doi.org/10.1159/000055366

31. Baldus SE, Zirbes TK, Hanisch FG, Kunze D, Shafizadeh ST, Nolden S, Monig SP, Schneider PM, Karsten U, Thiele J, Holscher AH, Dienes HP (2000) Thomsen-Friedenreich antigen presents as a prognostic factor in colorectal carcinoma: a clinicopathologic study of 264 patients. Cancer 88(7):1536-1543 
32. Takanami I (1999) Expression of Thomsen-Friedenreich antigen as a marker of poor prognosis in pulmonary adenocarcinoma. Oncol Rep 6(2):341-344

33. Hirao T, Sakamoto Y, Kamada M, Hamada S, Aono T (1993) Tn antigen, a marker of potential for metastasis of uterine cervix cancer cells. Cancer 72(1):154-159

34. Choufani G, Nagy N, Saussez S, Marchant H, Bisschop P, Burchert M, Danguy A, Louryan S, Salmon I, Gabius HJ, Kiss R, Hassid S (1999) The levels of expression of galectin-1, galectin-3, and the Thomsen-Friedenreich antigen and their binding sites decrease as clinical aggressiveness increases in head and neck cancers. Cancer 86(11):2353-2363
35. Heimburg J, Yan J, Morey S, Glinskii OV, Huxley VH, Wild L, Klick R, Roy R, Glinsky VV, Rittenhouse-Olson K (2006) Inhibition of spontaneous breast cancer metastasis by anti-ThomsenFriedenreich antigen monoclonal antibody JAA-F11. Neoplasia 8(11):939-948. https://doi.org/10.1593/neo.06493

36. Egger M (2014) Untersuchungen zur Expression von Mucin1 im Tumorgewebe von Brustkrebspatientinnen: eine Studie zur Korrelation mit dem Thomsen-Friedenreich Antigen und mit Her4 sowie Phospho-Her4. Dissertation. LMU München: Medizinische Fakultät

\section{Affiliations}

\section{Sabine Heublein ${ }^{1,2} \cdot$ Markus Egger $^{2,3} \cdot$ Junyan $\mathrm{Zhu}^{2} \cdot$ Luisa Berger $^{2} \cdot$ Doris Mayr $^{4} \cdot$ Christian Schindlbeck $^{5}$. Christina Kuhn ${ }^{2}$. Simone S. Hofmann ${ }^{2}$. Florian Schuetz ${ }^{1}$ Udo Jeschke ${ }^{2,6} \cdot$ Nina Ditsch $^{2,6}$}

Markus Egger

markus.egger@stanna.at

Junyan Zhu

zhu.junyan@med.uni-muenchen.de

Luisa Berger

luisaberger96@gmail.com

Doris Mayr

doris.mayr@med.uni-muenchen.de

Christian Schindlbeck

christian.schindlbeck@kliniken-sob.de

Christina Kuhn

christina.kuhn@med.uni-muenchen.de

Simone S. Hofmann

simone.hofmann@med.uni-muenchen.de

Florian Schuetz

florian.schuetz@med.uni-heidelberg.de

Udo Jeschke

udo.jeschke@med.uni-muenchen.de

Nina Ditsch

nina.ditsch@med.uni-muenchen.de
Department of Obstetrics and Gynecology, Heidelberg University Hospital, Heidelberg, Germany

2 Department of Obstetrics and Gynecology, Ludwig-Maximilians-University of Munich, Munich, Germany

3 St. Anna Kinderspital, Vienna, Austria

4 Department of Pathology, Ludwig-Maximilians-University of Munich, Munich, Germany

5 Klinikum Traunstein, Traunstein, Germany

6 Department of Obstetrics and Gynecology, University Hospital Augsburg, Augsburg, Germany 